ACTA AGROBOTANICA

Vol. 58, z. 12005

s. 3743

\title{
Seed treatment for control leaf spot diseases of spring wheat
}

\author{
BARBARA KRZYZIŃSKA, MARIOLA GEAZEK, \\ AGNIESZKA MĄCZYŃSKA
}

Plant Protection Institute, Sosnicowice Branch, ul. Gliwicka 29, 44153 Sosnicowice

(Received: 15.03.2005)

S u m m a r y

In the years 2001 and 2002 at the Institute of Plant Protection Branch in Sośnicowice research work was performed on seed treatment with fungicides against leaf diseases of spring wheat cv. Ismena caused by pathogenic fungi: Blumeria graminis, Phaeasphaeria nodorum, Puccinia recondita i Pyrenophora tritici-repentis. Two variants of protection were included in the experiment: seed dressing with fungicides or seed dressing and single application of foliar spray at GS 49.

At early growth stages of spring wheat a very high biological activity against powdery mildew, septoria leaf spot, brown rust and tan spot was recorded for seed dressing containing triticonazole+prochloraz. In the case of triadimenol+imazalil+fuberidazole only a weak, but long-lasting effect against brown rust and septoria leaf spot was observed. Leaf infection as well as 1000 grain mass and yield, in the experimental combination with using triticonazole+prochloraz were on the same level as in the experimental variant where after seed dressing with triadimenol+imazalil+fuberidazole plots were sprayed with tridemorf+epoxykonazole at GS 49. It was concluded that seed dressing preparation containing triticonazole+prochloraz constituted a good base for protection of spring wheat against foliar diseases.

Key words: seed treatment, tritoconazole, triadimenol, prochloraz, leaf spot diseases

\section{INTRODUCTION}

Seed dressing is the easiest and the cheapest way of plant protection against seedborne pathogens, infection by pathogens present in the soil and plant debris. Seed dressing preparations can also confine to a considerable degree infection sources of other pathogens which cause, among others, fusarium foot rot, fusarium leaf spot, head scab, rust, septoria diseases, rhynchosporium scald and powdery mildew, as these pathogens easily infect delicate and defenceless plants at germination. Early development of cereals (growth, tillering, development of root system) has a crucial effect on plant condition and the amount of yield (Montfort 1996). In Poland cereals are 
cultivated on 70\% of arable land (Rocznik Statystyczny 2001). A high proportion of cereals in rotation favours the development of diseases. Seed dressing preparations are usually characterized by high a efficacy because of nearly $100 \%$ uniform coverage of kernels, and because of a wide range of protective action and a long-lasting activity they create optimal conditions for good development of plants. Only some seed dressing preparations can provide a long-lasting protection against leaf, stem base and ear pathogens. Spring cereals can be protected by seed dressings better than winter cereals because of much shorter by nearly half vegetation period (Petróczi et al. 2002).

Seed dressing preparation contains two biologically active ingredients: prochloraz and triticonazole in ten-fold larger proportion comparing to other seed dressings registrated for sale and application. Prochloraz is designed for plant protection at early growth stages against seedling blight, smuts, and also against pathogens causing stem base and leaf diseases. Triticonazole is a compound characterized by slow activation and movement through young plant parts thereby providing protection against fungal diseases for a very long time (Qu erou et al. 1998).

\section{MATERIALS AND METHODS}

Experiments were performed on the experimental field in Sośnicowice in the years 2001 and 2002, according to randomized block design at 4 replications on spring wheat $\mathrm{cv}$. Ismena.

The aim of the experiments was to estimate the efficacy of 2 seed dressing preparations against diseases caused by: Blumeria graminis, Phaeosphaeria nodorum, Puccinia recondita i Pyrenophora tritici-repentis. The experiments consisted of 2 combinations: seed dressing or seed dressing and foliar treatment with a fungicide at GS 49. Experimental data are presented in table 1.

Table 1.

Characterization of experiments

\begin{tabular}{|c|c|c|c|c|}
\hline No & $\begin{array}{c}\text { Growth stage of application T0-GS } 00 \\
\text { / Active ingredient }\end{array}$ & $\begin{array}{c}\text { Dose per } \\
100 \mathrm{~kg} \text { seeds }\end{array}$ & $\begin{array}{c}\text { Growth stage of application } \\
\text { foliar } \\
\text { T2-GS 49/ } \\
\text { Active ingredient }\end{array}$ & $\begin{array}{l}\text { Dose } \\
\text { per ha }\end{array}$ \\
\hline 1 & \multicolumn{4}{|l|}{ Untreated } \\
\hline 2 & triticonazole + prochloraz $(200+53.3 \mathrm{~g} / \mathrm{l})$ & $300+300 \mathrm{ml} \mathrm{H}_{2} \mathrm{O}$ & --- & --- \\
\hline 3 & triticonazole + prochloraz $(200+53.3 \mathrm{~g} / \mathrm{l})$ & $300+300 \mathrm{ml} \mathrm{H}_{2} \mathrm{O}$ & $\begin{array}{l}\text { tridemorf }+ \text { epoxykonazole } \\
(375+125 \mathrm{~g} / 1)\end{array}$ & $1.0 \mathrm{l} / \mathrm{ha}$ \\
\hline 4 & $\begin{array}{l}\text { triadimenol }+ \text { imazalil }+ \text { fuberidazole } \\
\qquad(75+10+9 \mathrm{~g} / \mathrm{l})\end{array}$ & $400+200 \mathrm{ml} \mathrm{H}_{2} \mathrm{O}$ & -- & --- \\
\hline 5 & $\begin{array}{l}\text { triadimenol }+ \text { imazalil }+ \text { fuberidazole } \\
\qquad(75+10+9 \mathrm{~g} / 1)\end{array}$ & $400+200 \mathrm{ml} \mathrm{H}_{2} \mathrm{O}$ & $\begin{array}{c}\text { tridemorf + epoxykonazole } \\
(375+125 \mathrm{~g} / 1)\end{array}$ & $1.0 \mathrm{l} / \mathrm{ha}$ \\
\hline
\end{tabular}

Leaf infection by pathogens was estimated at GS 37, GS 55 and GS 75. At milky ripe stage (GS 75) green leaf area (GLA) was determined on flag leaves. The influence of leaf infection on grain yield was also studied. Results of two year experiments were averaged and presented as per cent of leaf area infection. Analysis of variance was performed at the level of probability $\mathrm{P}=0.05$. 


\section{RESULTS}

In vegetation seasons 2001 and 2002 brown rust and tan spot occurred, on average, in a considerable intensity, developing slowly in the spring and occupying several per cent of leaf blades and at later stages of plant growth over L of upper leaves area. Septoria leaf spot occurred in somewhat lower intensity occupying 8-9\% of leaf area. Powdery mildew more strongly infected spring wheat leaves that amounted to $11-16 \%$ of leaf area, and the infection could be considered as significant only at early growth stages of plants.

The results of estimation of leaf infection revealed that seed dressing preparation containing triticonazole+prochloraz showed a very high biological activity in confining the development of all four diseases till the end of shooting (GS 37). Second from used of seed dressing confined the development of diseases to a considerably lesser extent. At successive dates of disease estimation seed dressing preparation containing triticonazole+prochloraz also exhibited a confining effect on powdery mildew, tan spot, brown rust and septoria leaf spot. Leaf infection in this experimental combination was on the same level as stated for seed dressing containing triadimenol+imazalil+fuberidazole and a supplementary treatment of spring wheat plants at GS 49 with a fungicide. At milky ripe stage the effect of two ingredients seed dressing on powdery mildew and tan spot was somewhat lower, however in the case of brown rust and septoria leaf spot infection was on the same level as recorded in the experimental variant of seed treatment with triadimenol+imazalil+fuberidazole and a successive fungicide treatment at GS 49. From earing stage up to the end of vegetative period the confining effect of second from used seed dressing on leaf infection by Blumeria graminis and Pyrenophora tritici-repentis was not observed, only a weak influence on diminishing leaf infection by Puccinia recondita and Phaeosphaeria nodorum could be seen. Seed treatment preparation containing triticonazole+prochloraz more effectively confined the source of infection at earlier growth stages of spring wheat and thus provided a better protection at later growth stages of plants (table 2).

Analysis of variance confirmed the significance of reducing leaf infection and saving green leaf area both in the cases of seed treatment without or with an additional treatment of plants with a fungicide at GS 49. The only exception was seed treatment with triadimenol+imazalil+fuberidazole only, where no significant effect on saving green leaf area occurred (table 2, 3).

Seed dressing preparation containing triticonazole+prochloraz provided a better plant protection against spring wheat leaf diseases than second from used seed dressing. Seed dressing with triticonazole+prochloraz significantly contributed to the increase of 1000 grain mass $(3 \%)$ and grain yield (10\%). These parameters were on the same level as recorded for experimental variant where for seed dressing was used triadimenol+imazalil+fuberidazole and additional treatment of plants with a fungicide was performed. When seeds were treated with triticonazole+prochloraz and on additional single spray with a fungicide was applied, the increase of 1000 grain mass amounted to $15 \%$ and grain yield increased by $27 \%$. Only seed dressing with triadimenol+imazalil+fuberidazole had no significant effect on the increase of 1000 grain mass and yield (table 4). 
Table 2.

Influence of seed treatment and foliar application of fungicides on infection of spring wheat with foliar pathogens

\begin{tabular}{|c|c|c|c|c|c|c|}
\hline \multirow{2}{*}{ No } & \multirow{2}{*}{ Experimental objects } & \multirow{2}{*}{ Dose } & \multicolumn{4}{|c|}{$\%$ leaf infection } \\
\hline & & & GS 37 & GS 55 & GS 75 & Average \\
\hline \multicolumn{7}{|c|}{ Blumeria graminis } \\
\hline 1 & Untreated & --- & $11.05 \mathrm{~d}$ & $16.18 b$ & $8.75 \mathrm{c}$ & $11.99 \mathrm{~b}$ \\
\hline 2 & T0* triticonazole + prochloraz & T0 $300+300 \mathrm{ml} \mathrm{H}_{2} \mathrm{O}$ & $1.02 \mathrm{a}$ & $5.16 \mathrm{a}$ & $4.90 \mathrm{~b}$ & $3.70 \mathrm{a}$ \\
\hline 3 & $\begin{array}{l}\text { T0* triticonazole }+ \text { prochloraz } \\
\text { T2 } * * \text { tridemorf }+ \text { epoxykonazole }\end{array}$ & $\begin{array}{c}\mathrm{T} 0300+300 \mathrm{ml} \mathrm{H}_{2} \mathrm{O} \\
\text { T1 } 101 / \mathrm{ha} \\
\end{array}$ & $1.50 \mathrm{a}$ & $0.92 \mathrm{a}$ & $1.42 \mathrm{a}$ & $1.28 \mathrm{a}$ \\
\hline 4 & T0* triadimenol + imazalil + fuberidazole & $\mathrm{T} 0400+200 \mathrm{ml} \mathrm{H}_{2} \mathrm{O}$ & $8.43 \mathrm{c}$ & $11.55 b$ & $7.86 \mathrm{c}$ & $9.28 \mathrm{~b}$ \\
\hline 5 & $\begin{array}{l}\text { T0* triadimenol }+ \text { imazalil }+ \text { fuberidazole } \\
\text { T2** tridemorf }+ \text { epoxykonazole }\end{array}$ & $\begin{array}{l}\mathrm{T} 0400+200 \mathrm{ml} \mathrm{H}_{2} \mathrm{O} \\
\mathrm{T} 1 \quad 10 \mathrm{l} / \mathrm{ha}\end{array}$ & $3.78 b$ & $3.92 \mathrm{a}$ & $1.47 \mathrm{a}$ & $3.05 \mathrm{a}$ \\
\hline \multicolumn{3}{|c|}{$\operatorname{LSD}(0.05)$} & 2.11 & 530 & 157 & 281 \\
\hline \multicolumn{7}{|c|}{ Phaeosphaeria nodorum } \\
\hline 1 & Untreated & --- & $4.63 \mathrm{~d}$ & $9.06 \mathrm{~d}$ & $8.44 d$ & $7.37 \mathrm{~d}$ \\
\hline 2 & T0* triticonazole + prochloraz & $\mathrm{T} 0300+300 \mathrm{ml} \mathrm{H}_{2} \mathrm{O}$ & $0.12 \mathrm{a}$ & $2.37 \mathrm{~b}$ & $2.57 \mathrm{~b}$ & $1.68 \mathrm{ab}$ \\
\hline 3 & $\begin{array}{l}\text { T0* triticonazole }+ \text { prochloraz } \\
\text { T2 } * * \text { tridemorf }+ \text { epoxykonazole }\end{array}$ & $\begin{array}{c}\mathrm{T} 0300+300 \mathrm{ml} \mathrm{H} \mathrm{H}_{2} \mathrm{O} \\
\mathrm{T} 110 \mathrm{l} / \mathrm{ha}\end{array}$ & $0.61 \mathrm{ab}$ & $0.81 \mathrm{a}$ & $1.03 \mathrm{a}$ & $0.81 \mathrm{a}$ \\
\hline 4 & T0* triadimenol + imazalil + fuberidazole & $\mathrm{T} 0400+200 \mathrm{ml} \mathrm{H}_{2} \mathrm{O}$ & $2.48 \mathrm{c}$ & $7.36 \mathrm{c}$ & $5.52 \mathrm{c}$ & $5.12 \mathrm{c}$ \\
\hline 5 & $\begin{array}{l}\mathrm{T} 0 * \text { triadimenol }+ \text { imazalil }+ \text { fuberidazole } \\
\mathrm{T} 2 * * \text { tridemorf }+ \text { epoxykonazole }\end{array}$ & $\begin{array}{l}\mathrm{T} 0400+200 \mathrm{ml} \mathrm{H}_{2} \mathrm{O} \\
\mathrm{T} 110 \mathrm{l} / \mathrm{ha}\end{array}$ & $2.02 \mathrm{bc}$ & $2.25 \mathrm{ab}$ & $1.81 \mathrm{ab}$ & $2.03 b$ \\
\hline \multirow{2}{*}{\multicolumn{7}{|c|}{\begin{tabular}{|l|c|} 
LSD $(0.05)$ & 1.51 \\
& Pyrenophora tritici-repentis
\end{tabular}}} \\
\hline & & & & & & \\
\hline 1 & Untreated & --- & $1.42 \mathrm{~b}$ & $19.84 b$ & $25.52 \mathrm{~d}$ & $15.60 \mathrm{c}$ \\
\hline 2 & T0* triticonazole + prochloraz & T0 $300+300 \mathrm{ml} \mathrm{H}_{2} \mathrm{O}$ & $0.00 \mathrm{a}$ & $7.61 \mathrm{a}$ & $12.52 \mathrm{c}$ & $6.71 b$ \\
\hline 3 & $\begin{array}{l}\text { T0* triticonazole }+ \text { prochloraz } \\
\text { T2 } * * \text { tridemorf }+ \text { epoxykonazole }\end{array}$ & $\begin{array}{l}\text { T0 } 300+300 \mathrm{ml} \mathrm{H}_{2} \mathrm{O} \\
\text { T1 } 10 \mathrm{l} / \mathrm{ha}\end{array}$ & $0.00 \mathrm{a}$ & $1.25 \mathrm{a}$ & $5.99 \mathrm{a}$ & $2.41 \mathrm{a}$ \\
\hline 4 & T0* triadimenol + imazalil +fuberidazole & $\mathrm{T} 0400+200 \mathrm{ml} \mathrm{H}_{2} \mathrm{O}$ & $1.00 \mathrm{~b}$ & $21.83 \mathrm{~b}$ & $26.01 d$ & $16.27 \mathrm{c}$ \\
\hline 5 & $\begin{array}{l}\text { T0* triadimenol + imazalil +fuberidazole } \\
\text { T2** tridemorf }+ \text { epoxykonazole }\end{array}$ & $\begin{array}{l}\mathrm{T} 0400+200 \mathrm{ml} \mathrm{H} \mathrm{H}_{2} \mathrm{O} \\
\mathrm{T} 110 \mathrm{l} / \mathrm{ha}\end{array}$ & $0.71 \mathrm{ab}$ & $3.88 \mathrm{a}$ & $9.86 \mathrm{~b}$ & $4.81 \mathrm{ab}$ \\
\hline \multicolumn{3}{|c|}{ LSD (0.05) } & 0.89 & 651 & 2.40 & 252 \\
\hline \multicolumn{7}{|c|}{ Puccinia recondita } \\
\hline 1 & Untreated & --- & $5.59 \mathrm{c}$ & $15.80 \mathrm{c}$ & $28.44 d$ & $16.61 d$ \\
\hline 2 & T0* triticonazole + prochloraz & $\mathrm{T} 0300+300 \mathrm{ml} \mathrm{H}_{2} \mathrm{O}$ & $0.10 \mathrm{a}$ & $3.45 \mathrm{a}$ & $9.46 \mathrm{~b}$ & $4.34 \mathrm{~b}$ \\
\hline 3 & $\begin{array}{l}\mathrm{T} 0 * \text { triticonazole }+ \text { prochloraz } \\
\mathrm{T} 2 * * \text { tridemorf }+ \text { epoxykonazole }\end{array}$ & $\begin{array}{c}\mathrm{T} 0300+300 \mathrm{ml} \mathrm{H} \mathrm{H}_{2} \mathrm{O} \\
\mathrm{T} 110 \mathrm{l} / \mathrm{ha}\end{array}$ & $0.32 \mathrm{a}$ & $0.00 \mathrm{a}$ & $5.10 \mathrm{a}$ & $1.80 \mathrm{a}$ \\
\hline 4 & T0* triadimenol + imazalil + fuberidazole & T0 $400+200 \mathrm{ml} \mathrm{H}_{2} \mathrm{O}$ & $3.60 \mathrm{~b}$ & $10.54 b$ & $20.24 \mathrm{c}$ & $11.46 \mathrm{c}$ \\
\hline 5 & $\begin{array}{l}\text { T0* triadimenol }+ \text { imazalil }+ \text { fuberidazole } \\
\text { T2** tridemorf }+ \text { epoxykonazole }\end{array}$ & $\begin{array}{c}\mathrm{T} 0400+200 \mathrm{ml} \mathrm{H} \mathrm{H}_{2} \mathrm{O} \\
\mathrm{T} 110 \mathrm{l} / \mathrm{ha}\end{array}$ & $2.88 \mathrm{~b}$ & $3.34 \mathrm{a}$ & $6.21 \mathrm{ab}$ & $414 \mathrm{ab}$ \\
\hline LSD & $(0.05)$ & & 1.73 & 386 & 3.40 & 236 \\
\hline \multicolumn{7}{|c|}{ Sum $\%$ leaf infection } \\
\hline 1 & Untreated & --- & $22.68 \mathrm{~d}$ & $60.87 \mathrm{c}$ & $71.14 \mathrm{~d}$ & $51.56 \mathrm{~d}$ \\
\hline 2 & $\mathrm{~T} 0 *$ triticonazole + prochloraz & T0 $300+300 \mathrm{ml} \mathrm{H}_{2} \mathrm{O}$ & $1.25 \mathrm{a}$ & $18.58 \mathrm{~b}$ & $29.44 b$ & $16.42 b$ \\
\hline 3 & $\begin{array}{l}\text { T0* triticonazole }+ \text { prochloraz } \\
\text { T2 } * * \text { tridemorf }+ \text { epoxykonazole }\end{array}$ & $\begin{array}{c}\mathrm{T} 0300+300 \mathrm{ml} \mathrm{H} \mathrm{H}_{2} \mathrm{O} \\
\text { T1 } 10 \mathrm{l} / \mathrm{ha}\end{array}$ & $2.41 \mathrm{a}$ & $2.97 \mathrm{a}$ & $13.53 \mathrm{a}$ & $6.31 \mathrm{a}$ \\
\hline 4 & T0* triadimenol + imazalil +fuberidazole & $\mathrm{T} 0400+200 \mathrm{ml} \mathrm{H}_{2} \mathrm{O}$ & $15.50 \mathrm{c}$ & $51.25 \mathrm{c}$ & $59.62 \mathrm{c}$ & $42.13 \mathrm{c}$ \\
\hline 5 & $\begin{array}{l}\text { T0* triadimenol + imazalil +fuberidazole } \\
\text { T2** tridemorf }+ \text { epoxykonazole }\end{array}$ & $\begin{array}{c}\mathrm{T} 0400+200 \mathrm{ml} \mathrm{H}_{2} \mathrm{O} \\
\mathrm{T} 1 \quad 10 \mathrm{l} / \mathrm{ha}\end{array}$ & $9.38 b$ & $13.38 \mathrm{ab}$ & $19.34 \mathrm{a}$ & $14.03 \mathrm{~b}$ \\
\hline \multicolumn{3}{|c|}{$\operatorname{LSD}(0.05)$} & 5.68 & 13.57 & 5.90 & 767 \\
\hline
\end{tabular}

* T0 application of growth stage GS 00; ** T2 application of growth stage GS 49 
Table 3.

Influence of seed treatment and foliar application of fungicides on green leaf area of spring wheat GS 75

\begin{tabular}{|c|c|c|c|}
\hline No & Experimental objects & Dose & $\begin{array}{c}\text { Green leaf area } \\
\%\end{array}$ \\
\hline 1 & Untreated & --- & $34.45 \mathrm{a}$ \\
\hline 2 & T0 $*$ triticonazole + prochloraz & T0 $300+300 \mathrm{ml} \mathrm{H}_{2} \mathrm{O}$ & $46.06 \mathrm{~b}$ \\
\hline 3 & $\begin{array}{l}\text { T0* triticonazole }+ \text { prochloraz } \\
\text { T2** tridemorf }+ \text { epoxykonazole }\end{array}$ & $\begin{array}{c}\text { T0 } 300+300 \mathrm{ml} \mathrm{H}_{2} \mathrm{O} \\
\text { T1 } 1.0 \mathrm{l} / \mathrm{ha}\end{array}$ & $52.97 \mathrm{~b}$ \\
\hline 4 & T0* triadimenol + imazalil +fuberidazole & $\mathrm{T} 0400+200 \mathrm{ml} \mathrm{H}_{2} \mathrm{O}$ & $36.26 \mathrm{a}$ \\
\hline 5 & $\begin{array}{l}\text { T0* triadimenol }+ \text { imazalil }+ \text { fuberidazole } \\
\text { T2 } * * \text { tridemorf }+ \text { epoxykonazole }\end{array}$ & $\begin{array}{c}\mathrm{T} 0400+200 \mathrm{ml} \mathrm{H} \mathrm{H}_{2} \mathrm{O} \\
\mathrm{T} 11.0 \mathrm{l} / \mathrm{ha}\end{array}$ & $50.61 b$ \\
\hline \multicolumn{3}{|c|}{$\operatorname{LSD}(0.05)$} & 7.23 \\
\hline
\end{tabular}

Table 4.

Influence of seed treatment and foliar application of fungicides on average grain yield and weight of one thousand grains of spring wheat

\begin{tabular}{|c|c|c|c|c|c|c|c|c|}
\hline \multirow{3}{*}{ No } & \multirow{3}{*}{ Experimental objects } & \multirow{3}{*}{ Dose } & \multicolumn{3}{|c|}{$\begin{array}{c}\text { Average weight } \\
\text { of thousand grains }\end{array}$} & \multicolumn{3}{|c|}{ Average yield of grain } \\
\hline & & & \multirow[t]{2}{*}{ g } & \multicolumn{2}{|c|}{$\begin{array}{l}\text { Increase as } \\
\text { related to control }\end{array}$} & \multirow[t]{2}{*}{$\mathrm{t} / \mathrm{ha}$} & \multicolumn{2}{|c|}{$\begin{array}{l}\text { Increase as related } \\
\text { to control }\end{array}$} \\
\hline & & & & $\mathrm{g}$ & $\%$ & & $\mathrm{t} / \mathrm{ha}$ & $\%$ \\
\hline 1 & Untreated & --- & $27.24 \mathrm{a}$ & 0.00 & 100.00 & $2.68 \mathrm{a}$ & 0.00 & 10000 \\
\hline 2 & $\mathrm{~T} 0 *$ triticonazole + prochloraz & $\begin{array}{c}\text { T0 } 300+300 \mathrm{ml} \\
\mathrm{H}_{2} \mathrm{O}\end{array}$ & $28.15 b$ & 0.91 & 103.34 & $2.96 \mathrm{~b}$ & 0.29 & 110.65 \\
\hline 3 & $\begin{array}{l}\mathrm{T} 0 * \text { triticonazole }+ \text { prochloraz } \\
\mathrm{T} 2 * * \text { tridemorf }+ \text { epoxykonazole }\end{array}$ & $\begin{array}{c}\mathrm{T} 0300+300 \mathrm{ml} \\
\mathrm{H}_{2} \mathrm{O} \\
\text { T1 } 1.01 / \mathrm{ha} \\
\end{array}$ & $31.44 \mathrm{c}$ & 4.20 & 115.40 & $3.39 \mathrm{c}$ & 0.72 & 12682 \\
\hline 4 & $\begin{array}{l}\text { T0* triadimenol + imazalil } \\
\text { +fuberidazole }\end{array}$ & $\begin{array}{c}\mathrm{T} 0400+200 \mathrm{ml} \\
\mathrm{H}_{2} \mathrm{O}\end{array}$ & $27.25 \mathrm{a}$ & 0.01 & 100.04 & $2.76 \mathrm{a}$ & 0.09 & 103.27 \\
\hline 5 & $\begin{array}{l}\mathrm{T} 0 * \text { triadimenol }+ \text { imazalil } \\
+ \text { fuberidazole } \\
\mathrm{T} 2 * * \text { tridemorf }+ \text { epoxykonazole }\end{array}$ & $\begin{array}{c}\mathrm{T} 0400+200 \mathrm{ml} \\
\mathrm{H}_{2} \mathrm{O} \\
\mathrm{T} 11.0 \mathrm{l} / \mathrm{ha} \\
\end{array}$ & $29.61 b$ & 2.37 & 108.70 & $3.14 b c$ & 0.47 & 11738 \\
\hline LSD ( & 05) & & 0.90 & & & 026 & & \\
\hline
\end{tabular}

\section{DISCUSSION}

The only means of cereal protection against diseases transmitted with infected seeds and though the soil is seed dressing with fungicides. A chemical compound which would be effective against smuts, snow mould, barley leaf stripe and seedling blights when used for spraying plants in vegetative period is not known. Some biologically active systemic preparations, used both for plant spraying as seed dressings protect plants against diseases such as powdery mildew, brown rust and septoria infection (Russell et al. 1992; Wenz et al. 1998). Those are for example triticonazole, prochloraz and triadimenol. In experiments performed by La mari (2000) seed dressing preparation containing triadimenol+imazalil+fuberidazole confined at early growth stages the infection of wheat leaves by septoria leaf spot and powdery mildew by $40 \%$. Selected literature data indicate that triadimenol quite effectively decreases leaf infection by brown (Everst et Leath 1993) confining germination of spores and 
sporulation of this pathogen on leaves (Sundin et al. 1999). Formento (2002) observed long-lasting activity of triticonazole against septoria leaf spot and tan spot till the growth stage of flag leaf development. Data obtained by these authors are in agreement with the results obtained in experiments described in this paper. Seed dressing preparation containing triticonazole and prochloraz protected spring wheat plants against all studied pathogens almost up to the end of vegetative period. Similar results were obtained by Wen z (1998) who reports that protective action of selected seed dressings against leaf infecting pathogens such as brown rust and species of septoria can have a long-lasting effect. Results of experiments conducted by For me n to (2002) indicate that seed dressing preparations can protect leaves of cereals against fungal pathogens for 30 to 120 days, depending on content and type of biologically active compounds in plants from seeds to leaves starting at the early stage of plant development effectively retards infection with fungal diseases (Le ath and B owen 1989). According to Mugiera (1991) seed dressing can replace treatment of plants with fungicides in dependence on the degree of plant infection or make an excellent complementary element to foliar protection.

\section{REFERENCES}

Everts K. L., Leath S. 1993. Effect of triadimenol seed treatment and timing of foliar fungicide application on onset and extent of powdery mildew and leaf rust epidemics. Phytopathology 83: 557562.

Formento N. 2002. Eficacia de fungicidas para el tratamiento de semillas de trigo en siembra ditecta. EEA Inta Parano; 24: 256264.

Lamari L. 2000. Integrated management of leaf spot diseases in wheat. Project results ARDI 98 114; University of Manitoba; http:// www.gov.mb.ca/agriculture/research/ardi/pro jects/98 114.html: 19.

Leath L., B owen K. L. 1989. Effects of powdery mildew, triadimenol seed treatment, and triadimenol foliar sprays on yield of winter wheat in North Carolina. Phytopathology 79: 152155.

Montfort F. 1996. Effects of two triazole seed treatments, triticonazole and triadimenol, on growth and development of wheat. Pest. Sci. 46: 315322.

Mugier J., Chazalet M., Gatineau F. 1991. RPA 400727: Un nouveau fungicide pour le traitement des semences de cereals. ANNP; Troisieme, Conference Internationale sur les Maladies des Plantes, Bourdeaux 35 december 1991: 941948.

Petróczi M. I., Matuz J., Kótai C. 2002. Study of pesticide side effects in winter wheat trials. Acta Biologica Szegediensis; 46(3 4): 207208.

Querou R., Euvard M., Gauvrit C. 1998. Uptake and fate of triticonazole applied as seed treatment to spring wheat (Triticum aestivum L.). Pest. Sci. 53: 324332.

Russell P. E., Percival A., Coltman P. M., Green D. E. 1992. Fluquinconazole, novel broad spectrum fungicide for foliar application. The BCPC Conference Pests \& Diseases 2: 411418.

Rocznik Statystyczny Rzeczypospolitej Polskiej. 2001. Główny Urząd Statystyczny. Warszawa.

Sundin D. R., B ockus W. W., Eversmeyer M. G. 1999. Triazole seed treatments spore production by Puccinia recontida, Septoria tritici and Stagonospora nodorum from wheat leaves. Plant Disease, Vol. 83(4): 328332. 
Venz M., Russel P. E., Löchel A. M., Buschhaus H., Evans P. A., Bardsley E., Petit E., Puhl T. 1998. Seed treatment with fluquinconazole for control of cereal take all, foliar and seed borne diseases. The BCPC Conference Pests \& Diseases 3: 907912.

\title{
Zwalczanie chorób grzybowych liści za pomocą zapraw nasiennych w uprawie pszenicy jarej
}

\author{
Streszczen i e
}

W latach 2001-2002 w Instytucie Ochrony Roślin w Sośnicowicach prowadzono badania nad stosowaniem zapraw nasiennych w ochronie pszenicy jarej odmiany Ismena przed chorobami liści, których sprawcami są grzyby patogeniczne takie jak Blumeria graminis, Phaeasphaeria nodorum, Puccinia recondita i Pyrenophora tritici-repentis. W doświadczeniu zastosowano jako jedyną ochronę fungicydową zabieg zaprawiania ziarna siewnego lub zabieg zaprawiania oraz zabieg opryskiwania w fazie rozwojowej roślin GS 49.

We wczesnych fazach rozwojowych roślin pszenicy jarej zaprawa zawierająca tritikonazol+prochloraz wykazała bardzo wysoką aktywność biologiczna w ograniczeniu występowania mączniaka prawdziwego, septoriozy, rdzy i brunatnej plamistości liści. Nie obserwowano ograniczającego wpływu preparatu zawierającego triadimenol+imazalil+fuberidazol na porażenie liści pszenicy jarej przez grzyb Blumeria graminis i Pyrenophora tritici-repentis, jedynie wykazywał on słabe działanie w ochronie liści pszenicy jarej przed rdzą brunatną i septoriozą do końca wegetacji. W kolejnych obserwacjach, porażenie liści oraz uzyskane zwyżki masy tysiąca ziaren i plonu, w kombinacji doświadczalnej z zastosowaniem preparatu zawierającego prochloraz i tritikonazol było na poziomie zastosowania fungicydu w terminie T2 (GS 49) po zaprawie zawierającej triadimenol+imazalil+fuberidazol. Zaprawa zawierająca tritikonazol i prochloraz stanowiła także lepszą podstawę do dalszej ochrony roślin pszenicy jarej co miało odzwierciedlenie w uzyskaniu najwyższego plonu ziarna i masy tysiąca ziaren pszenicy jarej. 


\section{VACAT}

